\title{
Differential Rotation of the Sun from helioseismology and magnetic field study.
}

\author{
Elena Gavryuseva ${ }^{1}$ and Susanne Höfner ${ }^{2}$ \\ ${ }^{1}$ Institute for Nuclear Research of the Academy of Sciences of Russia, \\ Moscow, Russia \\ email: elena.gavryuseva@gmail.com
}

\begin{abstract}
Differential rotational rate of the large scale magnetic field and its temporal dependence has been evidenced at different latitudes through activity cycles 21-23. Rotational rate of the magnetic field at the latitudes above 55 degrees doesn't getting slower. Torsional waves with 11-year period are clearly seen in the magnetic field rotation. Rotation is slower where and when the magnetic field is getting stronger.

The time of the magnetic field emergence to the photosphere was estimated.

It was revealed the existence of quasi-stable over 30 years exceptionally regular and symmetric in the both hemispherest longitudinal structure. This structure is originated from the tachocline zone rotating like a rigid body with the rotation rate corresponding to the rotation in the photosphere on the 55-60 degrees of the helio-latitudes.
\end{abstract}

Keywords. Sun, magnetic field, rotation, longitudinal structure.

The measurements of the large scale solar magnetic field in the photosphere taken at Wilcox Solar Observatory since 1976 up to 2008 (http://wso.stanford.edu/synoptic.html) have been analyzed to deduce its latitudinal and longitudinal structures, its differential rotation, and their variability in time.

A longitudinal structure quasi-stable over 30 years exceptionally regular and symmetric in the both hemispheres has been found without any a priori assumption about the rotation of the Sun (Gavryuseva \& Godoli (2004), Gavryuseva (2006)). This structure is originated from the tachocline zone under the convective envelope.

Comparison between the rotational rate of the large scale magnetic field and helioseismological findings of omega as a function of latitude and radius was performed.

Differential rotational rate of the magnetic field and its temporal dependence has been evidenced at different latitudes through activity cycles (Gavryuseva \& Godoli (2004), Gavryuseva (2006), Gavryuseva (2007)). Rotational rate of the large scale magnetic field at the latitudes above 55 degrees doesn't getting slower. The time of emergence was estimated.

Torsional waves are clearly seen in the magnetic field rotation. Rotation is slower where and when the magnetic field is getting stronger.

Comparison between the rotation of the Sun inferred from helioseismology and the rotation of the large scale solar magnetic field permits to reveal that there are long living longitudinal structure originated from the tachocline zone rotating like a rigid body with the rotation rate corresponding to the rotation in the photosphere on the 55-60 degrees of the helio-latitudes. This longitudinal structure is clearly visible during present long interval of low solar activity. While during last cycles NN 21, 22 and 23 it was necessary to use the data set as long as 20 years about to select this regular longitudinal structure between the stochastic processes taken place in the photosphere. This study permits to combine the knowledge from helioseismology with solar activity phenomena ruled by the large scale magnetic field and to get a panoramic view on the internal structure and dynamics of the Sun. 


\section{References}

Gavryuseva, E., Godoli, G. 2004, Physics and Chemistry of the Earth, v. 31, iss. 1-3, pp. 68-76.

Gavryuseva, E. 2006, Solar Activity and its Magnetic Origin, Proceedings of the 233rd Symposium of the International Astronomical Union held in Cairo, Egypt, March 31 - April 4, ed. V. Bothmer; A.A. Hady. Cambridge University Press, pp. 61-62

Gavryuseva, E. 2006, ibid, pp. 65-66

Gavryuseva, E. 2007, AIP

Gavryuseva, E. 2007, AIP 